\title{
Experimental studies on binary water droplet collisions considering size ratio effects
}

\author{
Maohong Sui ${ }^{* 1}$, Martin Sommerfeld ${ }^{1}$, Lars Pasternak ${ }^{1}$ \\ ${ }^{1}$ Multiphase Flow Systems, Institute for Process Engineering, Otto-von-Guericke-University \\ Magdeburg; Hoher Weg 7b, D-06120 Halle (Saale), Germany \\ ${ }^{*}$ Corresponding author email: maohong.sui@ovgu.de
}

\begin{abstract}
The results of binary droplet collisions are usually bouncing, stretching/reflexing separation as well as coalescence and are usually summarized in so-called collision maps, where the impact parameter $B$ is plotted versus the Weber number. The outcome of droplet collisions depends on the fluid properties, droplet size ratio, the kinematics, and kinetic conditions. Such collision maps are used to delineate the different outcome scenarios by appropriate boundary lines that can be applied, for example, in numerical simulations. The present work focuses on the study of binary droplet collisions with defined size ratios and water of different quality (tap water and distilled water). Although water has been studied by many researchers, only droplets of the same size have been considered. However, size ratio effects have a great influence on the collision outcomes associated with a shift of the boundary lines normally towards higher Weber number [1]. For example in a spray, collisions of small droplets with larger ones are more likely to occur due to their different response behaviour [2]. Generally, binary droplet collision experiments were conducted by using two interacting droplet chains. But for obtaining experimentally also very small size ratio below 0.3 , an additional experimental arrangement was selected here. A droplet chain was injected into a spray. The spray droplets typically have a size distribution ranging up to about $200 \mu \mathrm{m}$ and the droplet generator produces droplet sizes between 600 and $700 \mu \mathrm{m}$. With these novel experiments it is now possible to extend and validate the boundary lines also including size ratio effects.
\end{abstract}

\section{Keywords}

Droplet collisions, shadow imaging, size ratio effects, water quality, collision map.

\section{Introduction}

A spray process is a very complex multiphase system in which various elementary processes are involved. In addition to the atomization and disintegration process of the droplets, the droplet size distribution is influenced by the collisions between droplets, with different possible outcomes. Typical outcomes of a collision between two droplets are bouncing, coalescence and separation and are plotted in the well-known collision maps $(B=f(W e)$ shown in Figure 1. In these maps the non-dimensional impact parameter $B$ (see Eq. 1) is plotted against collision Weber number (see Eq. 2), using however the size of the smallest participating droplet. However, the collision map depends on numerous droplet parameters, i.e., liquid properties of the droplets, droplet size ratio and kinematics as well as kinetic conditions. Therefore, an attempt is made to develop generalized correlations for the boundary lines between collision regimes that depend on the relevant dimensionless numbers. In the end, in addition to understanding the processes in the sprays, the structure of a generalized collision map is necessary for the prediction of sprays by the Euler/Lagrange approach [2], without the need to perform experiments whenever different fluid properties are considered.

During the last decades several models have been developed to predict the collision outcomes, mostly based on energy balances supported by experiments. The boundary between 
bouncing and coalescence/stretching separation can be identified by means of the experimental based model by Estrade et al. [3]. The models by Ashgriz and Poo [4], validated for equal-sized water droplets, are determining the lower boundaries for stretching separation and coalescence and the upper boundary of reflexive separation. The results from Qian and Law [5] presented ambient pressure effects on the collision maps of water and alkanes. The momentum theory-based model by Jiang et al. [6] accounts for viscosity effects in the location of the lower boundary line for stretching separation. Based on the Jiang et al. [6] model, Gotaas et al. [7] used experimental data from different glycol droplet collisions to specify values of $C_{a}$ and $C_{b}$, the constants used in this correlation. This model was improved by Sommerfeld and Pasternak [2] by finding a dependence between $C_{a}$ and the Ohnesorge number $O h$ and setting the other involved parameter $C_{b}=1$. The data from the experiments by Kuschel and Sommerfeld [8] as well as Sommerfeld and Kuschel [9] were obtained only with $\sim 380 \mu \mathrm{m}$ mono-sized droplets, however, for a multitude of different droplet liquids including water, alcohols, oils and polymer solutions. To further validate the 3 rd order polynomial fit of $C_{a}$ and Ohnesorge number, it was necessary to run experiments also with a variation of the droplet size ratio.

A modified model for the bouncing boundary line based on Estrade et al. [3] was developed by Sui and Sommerfeld [10]. In the experimental studies of Rabe et al. [11] and Foissac et al. [12] water droplet collision maps were presented, for different droplet size ratios, but only down to $\Delta=0.5$. As a basis for developing generalized boundary lines for both separation regimes a so-called symmetric Weber number was introduced [11]. Surprisingly, Rabe et al. [11] did not show any bouncing regimes for water droplets with all size ratios. The existence of bouncing however was clearly demonstrated in the well accepted studies of Qian and Law [5] as well as in the results presented by Kuschel and Sommerfeld [8]. It is astonishing that the same group (Foissac et al. [12]) also presented collision maps for water droplets with $\Delta=1.0$ clearly showing a bouncing region in the domain with large $B$ but not for small $B$ and small $W e$ (just as shown in Figure 1). However, compared to the studies of Rabe et al. [11] with $d_{s}=$ $450 \mu \mathrm{m}$, slightly smaller droplets were used, namely $300 \mu \mathrm{m}$. Also in a dusty environment the water droplet collision map was not strongly affected (Foissac et al. [12]). As revealed in the numerical analysis presented by Lain and Sommerfeld [1], the existence of a bouncing region and the extent of the bouncing region has a drastic effect on the coalescence rate and hence the predicted droplet size spectrum. Therefore, this issue needs further detailed experimental studies. In the next chapter the experimental methods are introduced. Numerous novel results are then presented for a range of droplet size ratios $(0.24<\Delta<0.91)$ and also comparing collision maps for distilled and tap water. The smallest realizable size ratio $\Delta \approx 0.24$ was already very close to the reported most likely existing collision size ratio in sprays [1].
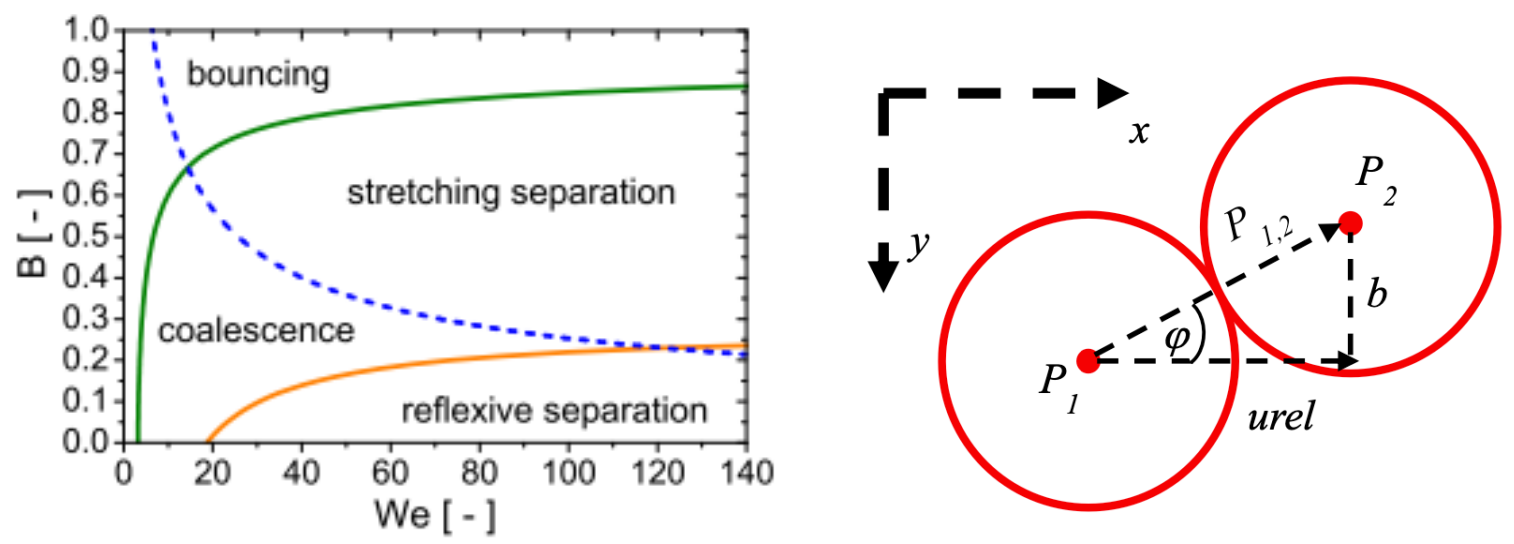

Figure 1. Typical droplet collision map showing the four droplet collision outcome scenarios and Droplet collision geometry for equal-sized droplets with relative velocity vector [9]. 


\section{Experimental setup}

\subsection{Droplet chain experimental facility}

For producing defined droplet-droplet collisions, the experiments were carried out using two oscillating membrane droplet generators (producer: Encap BioSystems, model: IE-0010H-P), each producing a chain of nearly equal spaced droplets. The water was supplied by a pressure vessel (see Figure 2). The droplet chains were generated by excitation of the liquid in the droplet generator and forced through different orifices of $100,200,300$ or $400 \mu \mathrm{m}$, respectively, which also resulted in different initial droplet sizes. The excitation frequency for breaking up the liquid jet depends on the size of the orifice and is between 500 and $4800 \mathrm{~Hz}$. The amplitude of the excitation signal was adjusted via an amplifier. Two high-speed cameras were mounted on the traversing system, with the front camera recording the drop impact scenarios, while the other synchronized high-speed camera was aligned parallel to the impact plane to record any eccentric impacts that were excluded from further analysis. The relative velocity, and consequently the Weber number, was adjusted by changing the angle of the droplet chains by rotation stages for each droplet generator, while the impact parameter B was changed by using the aliasing or frequency shifting method. (Gotaas et al. [7]).

As described above, the droplet size strongly depends on the flow rate, the size of the orifice, and the excitation frequency generated by the amplifier. In experimental cases with small size ratio $\Delta$, the smaller droplet chain needs a smaller size nozzle driven at higher frequency, therefore, the spacing between droplets becomes relatively small. Moreover, the velocity magnitude of the larger droplet chain is smaller than that of the smaller droplets. Thus, the larger droplets would hit more than one small droplet before the collision outcome has developed and is detectable. Furthermore, binary collision with low $B$ and with small size ratio would be even harder to be observed. Therefore, additionally another experimental set up was used, where a droplet chain was directed into a spray issuing from the pressure-driven nozzle. Illumination of the collision process was provided by two backlight LED arrays. In combination with the lens and an extension tube, the camera resolution was eventually $16.6 \mu \mathrm{m} / \mathrm{pixel}$ for the main front high-speed cameras.

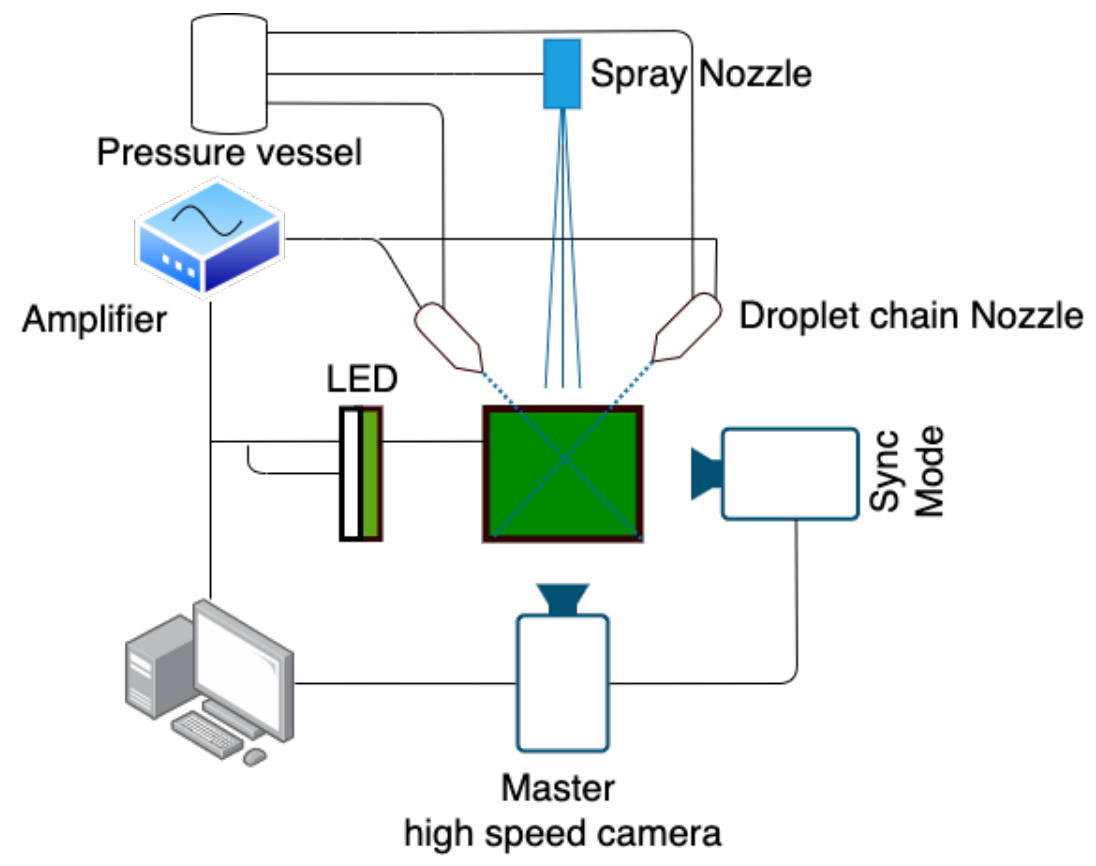

Figure 2. Sketch of the experimental setup with droplet chains and spray nozzle, liquid supply from a pressure vessel, LED illumination and high-speed camera recording as well as image recording PC. 


\subsection{Droplet chain interacting with a Spray}

For obtaining even smaller size ratios, lower than 0.3 , a novel experimental set-up was realized by using a spray nozzle for producing the smaller droplets and directing the chain of larger droplets produced by the droplet generator through the spray. Naturally with this arrangement the collisions of droplets occurred randomly. The one-fluid pressure nozzle (SCHLICK-Mod.553; 0.5 Liter/Min at 3 bar) was operated with 2.5 bar air pressure for producing a droplet size spectrum between 20 and $280 \mu \mathrm{m}$.

However, if the spray nozzle outlet is located close to the droplet chain, the entrained air will generate high velocities with strong turbulence, thus destroying and disturbing the droplet chain path. As a result, many chain droplets will not pass through the thin focal plane of the optical system. Another problem is that several collisions may occur within the dense spray area on a single image, which is then difficult to analyze automatically. In the end, the spray nozzle exit was placed father away from the droplet chain trajectory as illustrated in Figure 2. Consequently, collisions between chain droplets and finer spray droplets are statistically not very frequently occurring. Applying a frame rate of 10,000 images/s for the high-speed cameras, an image size of 702 pixels by 512 pixels is captured, yielding with the magnification of $16.6 \mu \mathrm{m} / \mathrm{pixel}$ an actual observation area size of $11.2 \mathrm{~mm}$ by $8.49 \mathrm{~mm}$. However, the radius of the spray at the considered downstream location is around $40 \mathrm{~cm}$. Therefore, the number of the small droplets that can be captured on the main high-speed camera is really small. Therefore, it was necessary to evaluate a large number of images for obtaining the results for small droplet size ratios.

The data-rate of droplet chain experiment is approximately $1.5 \%$. However, the data-rate of the spray case is roughly $0.056 \%$, which the data-rate is calculated by the number of detected collisions vs the number of total amount images.

\subsection{Repeatability of the experiments}

For the setup of Oscillating membrane droplet generators, the experiments have been done since 2012, as the paper published by Kuschel and Sommerfeld [8]. As to the second setup of spray and Oscillating membrane droplet generator, with fixed working conditions and relative location of spray generator and droplet chain generator. It can be repeated but requires a lot of post-processing work as mentioned before.

\section{Image Processing}

3.1 Droplet/Object detection

All images are first applied to a background subtraction and a binarization. Then image processing is continued with a LOG-filter (Laplacian of Gaussian) for sharpening and detecting the contours or interfaces of droplets or liquid fragments. Only in-focus objects are considered for further analysis, e.g., determine the cross-section area and finally the size of spherical droplets as well as the centroid location which is needed for tracking the droplets. All out-off focus and blurred objects are removed from the images. Consequently, the error of droplet location and size detection is less than one pixel.

\subsection{Object tracking}

Two object tracking tools were used for the two different experimental setups considered. The first approach (P1) uses an inhouse script of ImageJ, descripted in Pasternak and Sommerfeld [13] for droplet chain collisions. The second approach (P2) is based on a similar idea but generated by OpenCV [14] with the workflow as shown in Figure 3. The difference between these two approaches is the droplet tracking and collision detection part. The P1 was designed for oscillating membrane droplet generators facilities, a target 
droplet is selected and tracked to look for its collision partner coming from the other droplet chain. Then, while a drop-drop collision is detected and identified, backtracking of the collision partner is performed to determine the position of the collision partner in each previous frame before collision. Therefore, the velocity of the collision partner is calculated by its displacement vs travelling time. In this method, the collision partner's trajectory must be predictable, otherwise, the tracing-back is not working properly.

However, in the spray-droplet case, the collision partners have random trajectories and may come from anywhere above the droplet chain. Therefore, another image processing approach (P2), inspired by the vehicle detection on highways, was applied using OpenCV. In this approach, all well focused droplets are tracked and labeled in each frame and then in the next step connected as candidates for collisions. Afterwards, collisions would be detected by hand and recorded by labels of collision pairs.

\subsection{Data analysis}

For all recorded collision pairs with centroids, sizes, velocities and contact location $W e$ and $B$ are calculated. In both approaches, the positions and velocities from the droplets are based on their center of mass. Due to the fluctuations of the instantaneous velocities, each collision sequence, consists of at least six images before the first contact. Out of the instantaneous data the mean droplet velocities are calculated and the droplet contact point is estimated by the velocities and the last droplet position before contact. The contact point position is used to calculate the non-dimensional impact parameter $B$, which describes the geometry of the collision. The impact parameter is the sinus of the enclosed angle between the relative velocity and the position vector between the droplet center points. The mean droplet relative velocity and diameter are used for the other non-dimensional numbers: The Weber number $W e$, size ratio $\Delta$ (see Eq. 4) and the droplet Reynolds number $R e$ (see Eq. 5). The effect of the viscosity is included in the Ohnesorge number $O h$.

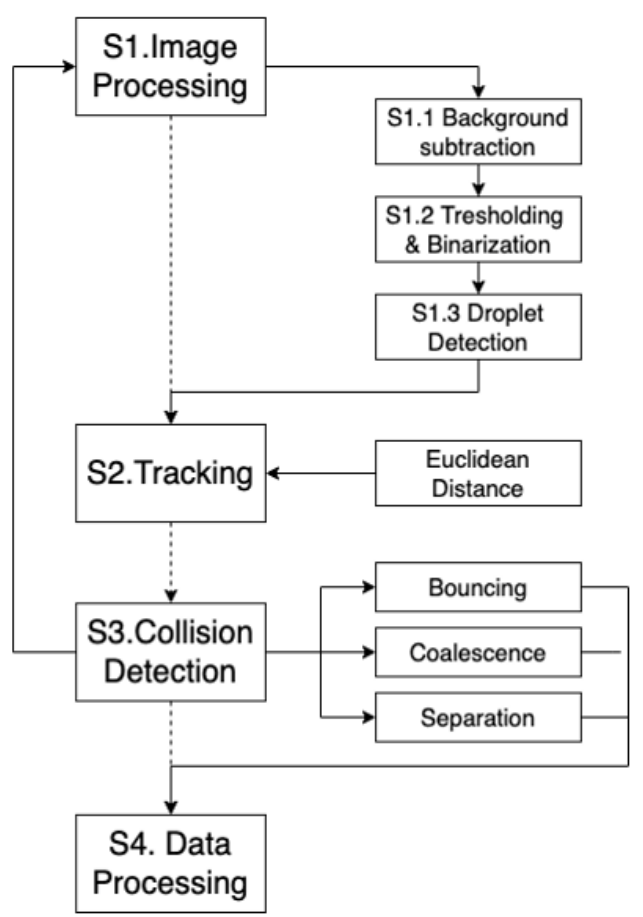

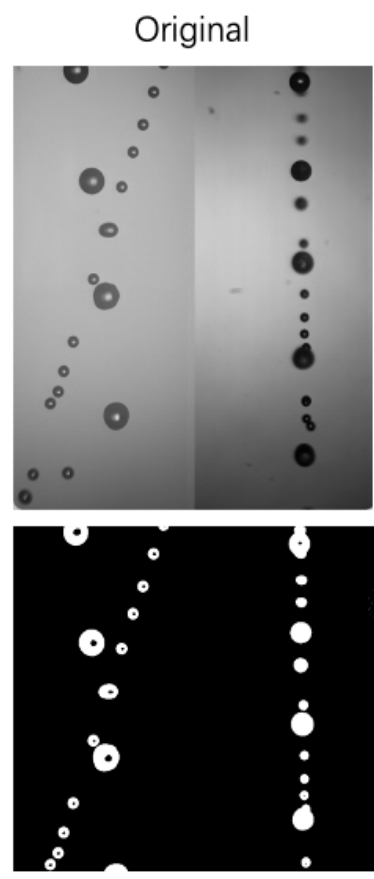

S1.2
S1.1
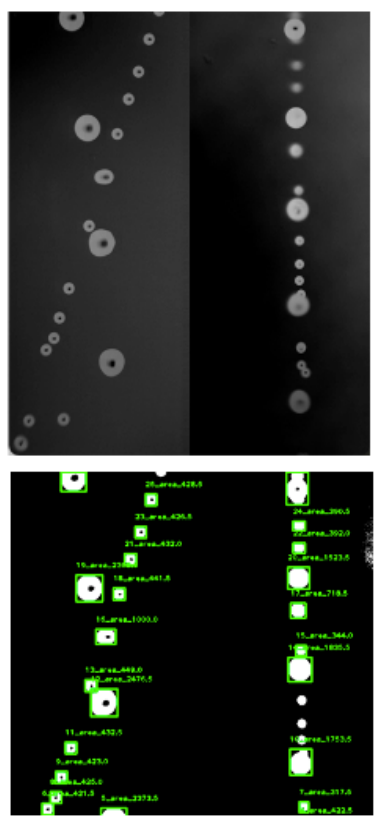

S1.3

Figure 3. Image processing flow chart and example of droplet chain collision with the processing approach P2. 


\section{Collision Map and boundary lines}

For the collision outcome classification, several theoretical boundaries are considered, namely the models of Estrade et al. [3] (see Eq. 6), Ashgriz and Poo [4] (see Eq. 7a and 7b) as well as the combined model for the coalescence-stretching separation boundary by Sommerfeld and Pasternak [2]. As suggested, in the Jiang et al. [6] correlation the parameters are $C_{b}=1$ and $C_{a}$ is represented by polynomial equation for pure liquids (see Eq. 8, 9). The measured properties of distilled water are: $\rho=998\left[\frac{\mathrm{kg}}{\mathrm{m}^{3}}\right], \sigma=68.8\left[\frac{\mathrm{mN}}{\mathrm{m}}\right], \mu=0.735[\mathrm{mPas}]$, and tap water: $\rho=997\left[\frac{\mathrm{kg}}{\mathrm{m}^{3}}\right], \sigma=65\left[\frac{\mathrm{mN}}{\mathrm{m}}\right], \mu=0.755[\mathrm{mPas}]$.

$$
\begin{aligned}
& B=\frac{2 b}{d_{1}+d_{2}}=\sin \phi \\
& W e=\frac{\rho d_{s} u_{r e l}^{2}}{\sigma} \\
& O h=\frac{\mu}{\sqrt{\rho \sigma d_{s}}} \\
& \Delta=\frac{d_{s}}{d_{l}} \\
& \operatorname{Re}=\frac{\sqrt{W e}}{O h}=\frac{\rho d_{s} u_{r e l}}{\mu}
\end{aligned}
$$

Estrade[3] Model for

Bouncing:

$$
\begin{gathered}
W e=\frac{\Delta\left(1+\Delta^{2}\right)\left(4 \phi^{\prime}-12\right)}{\chi(\cos (\arcsin B))^{2}} \quad \text { with } \phi^{\prime}=3.351 \\
W e=\frac{4\left(1-\Delta^{3}\right)\left[3(1+\Delta)(1-B)\left(\Delta^{3} \phi_{s}+\phi_{l}\right)\right]^{\frac{1}{2}}}{\Delta^{2}\left[\left(1+\Delta^{3}\right)-\left(1-B^{2}\right)\left(\phi_{s}+\Delta^{3} \phi_{l}\right)\right]} \\
W e_{c}=3\left[7\left(1+\Delta^{3}\right)^{\frac{2}{3}}-4\left(1+\Delta^{2}\right)\right] \frac{\Delta\left(1+\Delta^{3}\right)^{2}}{\Delta^{6} \eta_{s}+\eta_{l}}
\end{gathered}
$$

Combined model [2]: $\quad B=\frac{C_{a}}{1.14 W e^{\frac{1}{2}}}\left[1+\frac{\mu}{\sigma}\left(\frac{\rho d_{s}}{\sigma}\right)^{\frac{1}{2}}\right]\left[\left(\frac{1}{\Delta}\right)^{3}-2.4\left(\frac{1}{\Delta}\right)^{2}+2.7\left(\frac{1}{\Delta}\right)\right]^{\frac{1}{2}}$

Table 1. Summary of conducted experiments with droplet sizes in each case and Ohnesorge number calculated with the small droplet diameter.

\begin{tabular}{|c|c|c|c|c|c|}
\hline Water Type & Case & $d_{s}[\mu \mathrm{m}]$ & $d_{l}[\mu \mathrm{m}]$ & $\Delta$ & $O h_{s}[-]$ \\
\hline \multirow{4}{*}{ Distilled water } & Case 1 (Spray) & $130 \sim 180$ & $600 \sim 700$ & $\sim 0.24$ & 0.00717 \\
\cline { 2 - 6 } & Case 2 & 276 & 789 & 0.35 & 0.00534 \\
\cline { 2 - 6 } & Case 3 & 388 & 879 & 0.44 & 0.00450 \\
\cline { 2 - 6 } & Case 4 & 410 & 810 & 0.51 & 0.00438 \\
\cline { 2 - 6 } & Case 5 & 484 & 739 & 0.66 & 0.00403 \\
\cline { 2 - 6 } & Case 6 & 490 & 700 & 0.7 & 0.00401 \\
\cline { 2 - 6 } & Case 7 & 567 & 697 & 0.81 & 0.00373 \\
\hline \multirow{2}{*}{ Tap Water } & Case 8 & 473 & 674 & 0.7 & 0.00408 \\
\cline { 2 - 6 } & Case 9 & 650 & 713 & 0.91 & 0.00348 \\
\hline
\end{tabular}

\section{Results and Discussion}

The collision maps $B=f(W e)$ are approved to describe the collision outcome of spray droplet collision processes for certain conditions. The following collision maps (see Figure 4) show the results for distilled and tab water with the variation of absolute droplet size and size ratios. The experiments were performed up to a Weber number of about 50 . Higher Weber numbers 
could not be reached otherwise the droplets would not be spherical in the air. The collision map for $\Delta=0.24$ was generated with the droplet chain penetrating into the spray. Here the size of the collision pairs is more difficult to control than in the collision experiments with oscillating membrane droplet generators. The other collision maps presented, i.e. $\Delta=0.35$, $0.44,0.51,0.66,0.7$ and 0.81 are generated by oscillating membrane droplet generators with controlled droplet sizes.

Firstly, it can be noted that in the present experiments, regardless of the size ratio, bouncing of droplets was observed for larger $B$, which usually covers the whole range of $W e$. This finding is in agreement with the experiments of Kuschel and Sommerfeld [9] as well as Qian and Law [5] although in this study bouncing seems to extend in some cases down to smaller B-values. In the paper of Rabe et al. [11], no bouncing region was reported although in the present study similar droplet sizes were considered. Compared to the case of water droplet collisions in a 1 bar air atmosphere given by Qian and Law [5], the bouncing regime can be found in this study at $W e<5$ with $B>0.6$. This point is the triple point in the paper of Qian and Law [5] between bouncing and coalescence regime. Moreover, in Case 7, it is confirmed that the bouncing may happen also for B down to about 0.4 .

Next the effect of droplet size ratio on the structure of the collision maps will be discussed. First, as the size ratio increases, the regime of reflexive separation moves into the diagram (from $=0.51$ ) and the critical Weber number continuously decreases. In the cases with a small size ratio, i.e. $<0.6$, the regime of stretching separation is only observed in a very small region with higher B. However, in case 1 (spray Case) no clear boundary can be drawn between stretching separation and bouncing as well as coalescence. For this condition, more experimental data should be collected. In accordance with previous studies (Sommerfeld and Pasternak [13]), with increasing size ratio, the regime of stretching separation is expanding (i.e. the stretching separation-coalescence, boundary is moved downwards) and the triple point is shifted to the left, i.e. to smaller $W e$. As a result, the coalescence regime naturally shrinks with increasing size-ratio, which is the same trend as in the collision maps reported by Rabe et al. [11]. During the analyses of the droplet collisions in the spray case, it became obvious that collisions with large droplet size ratios are more dynamic and accompanied by large deformations of both droplets (examples are shown in Figure 5). On the other hand, the small droplets are being more easily absorbed by the larger ones, i.e. the small droplets most likely penetrate into the large droplets. Therefore, the oscillation of the combined droplet mass is not that pronounced and rapidly decays.

As to the boundary lines. First of all, the standard Estrade et al. [3] model with shape factor 3.351 is only shown in the collision maps for clarity (Figure 4). Additional measurements are also needed for larger Weber-numbers for identifying the lower bouncing boundary (i.e. We > 50). However, based on the results shown here, the prediction of the Estrade model for the bouncing region at higher We performs not very well and is located far below the experimentally indicated boundary. The Ashgriz and Poo [4] model for the SSC (stretching separation/coalescence) boundary has the problem that it is turning around the point (We:B = $0: 1$ ) with decreasing size ratio as stated by Sommerfeld and Pasternak [1]. However, the suggested combined model [1] for the boundary between coalescence and stretching separation shows excellent agreement with the present new measurements until the location of the triple point. As already discussed by Sommerfeld and Pasternak [1] below a size ratio of 0.5 the combined approach which includes the model of Brazier-Smith et al.[15] does not properly capture the size ratio effect. Nevertheless, the Ashgriz and Poo [4] model is included here as a reference although it is always below the experimentally found stretching separation/coalescence boundary. 


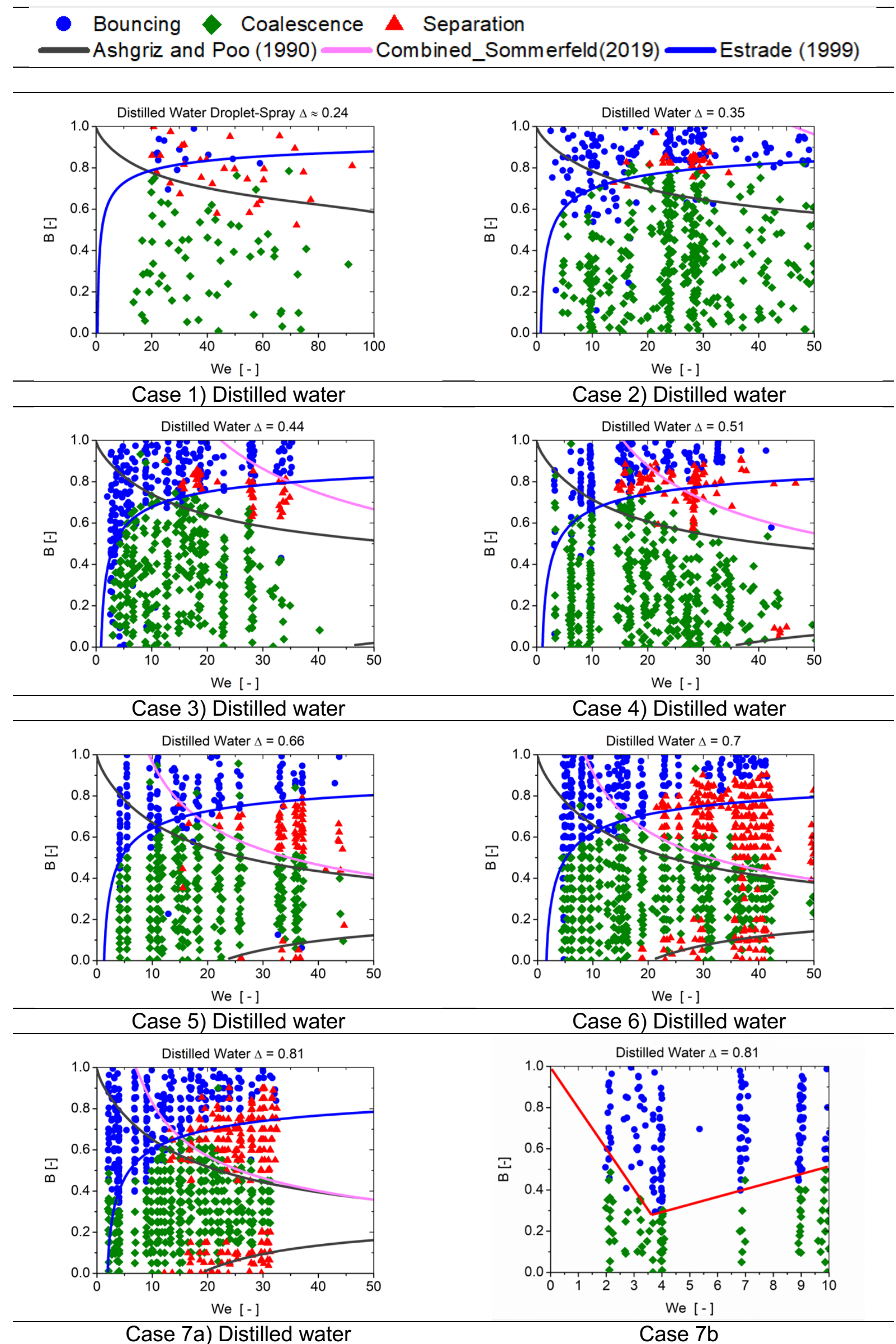

Case 7a) Distilled water

Case $7 \mathrm{~b}$ 


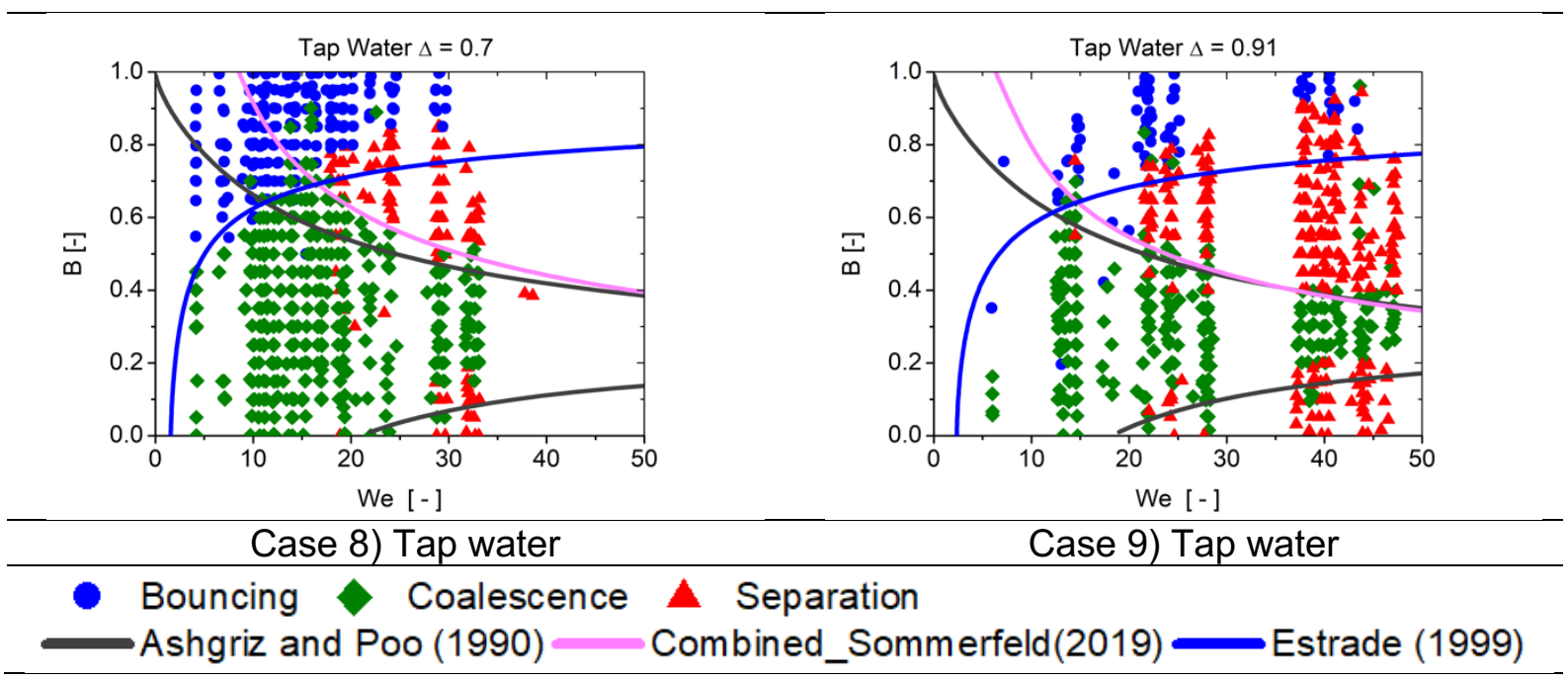

Figure 4. Collision maps for water droplets with different size ratio and different water quality; including the boundary lines of Estrade et al. [3], Ashgriz and Poo [4] as well as the combined model of Sommerfeld and Pasternak [2].

The influence of water quality may be identified by comparison between Case 6 and Case 8 . The collision maps are very similar, indicating that the water quality seems to have almost no effect on the collision maps.

Some examples of the collision scenarios and outcomes for different droplet size ratios and for different combinations of impact parameter and Weber number are summarized in Figure 5. These image series may be also connected to a single point in the collision maps as shown in Figure 4.

\section{Conclusions}

For the first time the effect of droplet size ratio on the outcome of water droplets binary collisions was investigated in detail and collision maps are presented. This is especially important as water sprays are often considered as reference case. All the collision maps shown in this paper have a bouncing regime which spans over all Weber numbers (measurements were conducted for $\mathrm{We}<50$ ) at larger impact parameters (i.e. B > 0.8). However, also at smaller Weber numbers (i.e. We $<5-10$ ) bouncing was observed down to about $B \approx 0.5$. This is conform with the measurements of Qian and Law [5], Foissac et al. [12] as well as Kuschel and Sommerfeld [8]. To clarify this issue additional measurements at very low We need to be conducted. Moreover, the collision maps need to be extended for larger Weber number $(\mathrm{We}>50)$ to further elaborate the lower bouncing boundary developed by Sui et al. [10].

The present studies clearly revealed that with reducing droplet size ratio for water the domain of coalescence is continuously growing. This is caused by the right-moving of reflexive separation to higher We, as well as the upwards shift of the SSC (stretching separation/coalescence) boundary. In addition, when reducing droplet size ratio, stretching separation is also slightly shifted to the right, to larger We. It is shown that also for water the combined model proposed by Sommerfeld and Pasternak [1] very well predicts the SSC boundary until the triple point for larger size ratios, i.e. $\Delta>0.5$. For smaller size ratio the theory of Brazier-Smith et al [15] needs to be revised. Moreover, the lower bouncing boundary line according to Sui et al.[10] will be updated with respect to the size ratio effect analyzed here. The water quality seems to have not a large effect on the collision maps although surface tension is slightly lower for tap water. 


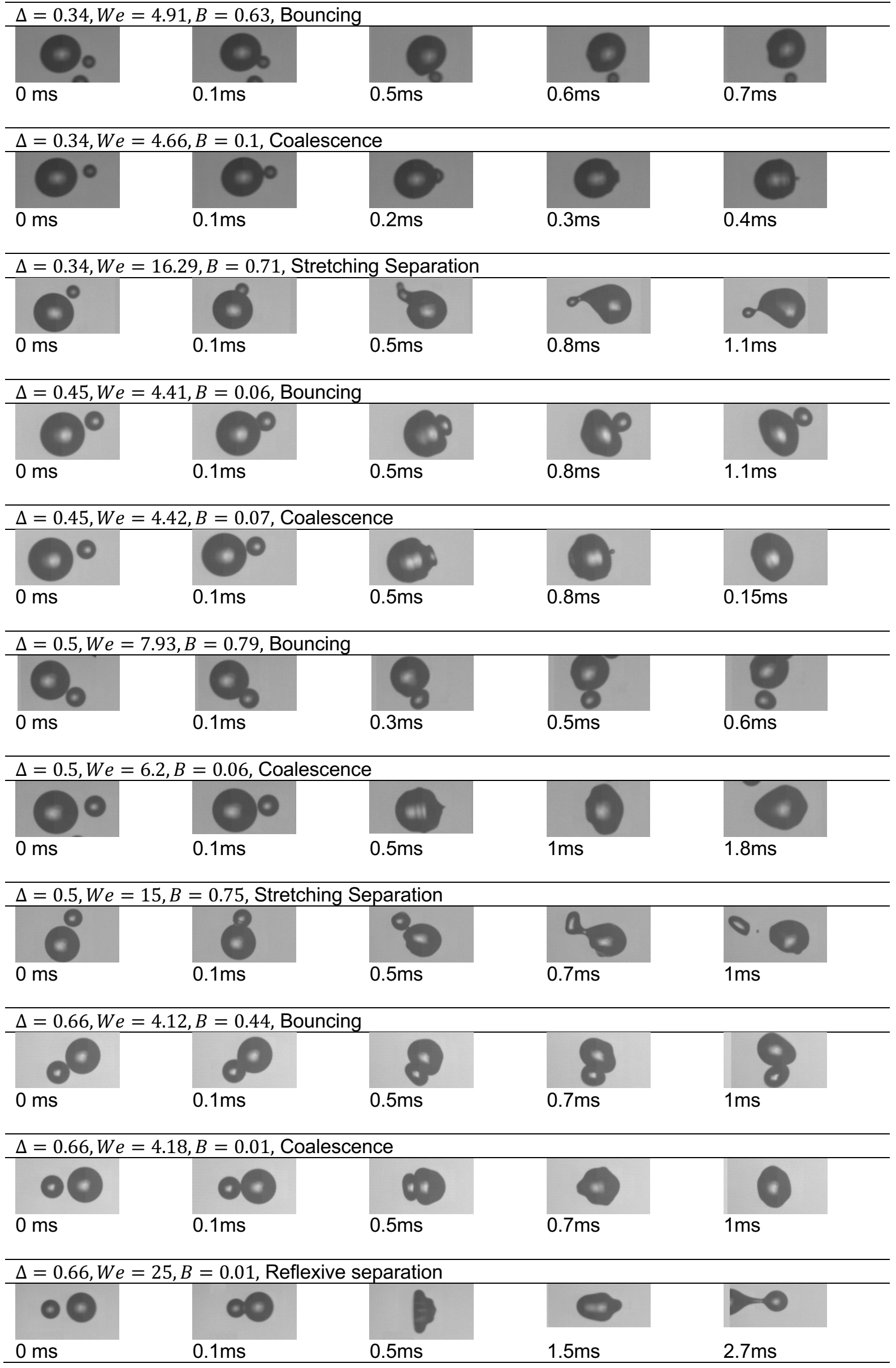




\begin{tabular}{|c|c|c|c|c|}
\hline \multicolumn{5}{|c|}{$\Delta=0.66, W e=14.91, B=0.76$, Stretching Separation } \\
\hline $0 \mathrm{~ms}$ & $0.1 \mathrm{~ms}$ & $0.5 \mathrm{~ms}$ & $0.7 \mathrm{~ms}$ & $1 \mathrm{~ms}$ \\
\hline \multicolumn{5}{|c|}{$\Delta=0.72, W e=9, B=0.59$, Bouncing } \\
\hline $0 \mathrm{~ms}$ & $0.1 \mathrm{~ms}$ & $0.5 \mathrm{~ms}$ & $0.7 \mathrm{~ms}$ & $0.9 \mathrm{~ms}$ \\
\hline \multicolumn{5}{|c|}{$\Delta=0.72, W e=19, B=0$, Reflexive separation } \\
\hline $0 \mathrm{~ms}$ & $0.1 \mathrm{~ms}$ & $0.5 \mathrm{~ms}$ & $1.2 \mathrm{~ms}$ & $3.4 \mathrm{~ms}$ \\
\hline \multicolumn{5}{|c|}{$\Delta=0.8, W e=3.75, B=0.29$, Bouncing } \\
\hline $0 \mathrm{~ms}$ & $0.1 \mathrm{~ms}$ & $0.5 \mathrm{~ms}$ & $1 \mathrm{~ms}$ & $1.7 \mathrm{~ms}$ \\
\hline \multicolumn{5}{|c|}{$\Delta=0.8, W e=3.75, B=0.29$, Coalescence } \\
\hline $0 \mathrm{~ms}$ & $0.1 \mathrm{~ms}$ & $0.5 \mathrm{~ms}$ & $1 \mathrm{~ms}$ & $1.5 \mathrm{~ms}$ \\
\hline
\end{tabular}

Figure 5. Illustrations of collision sequences for different droplet size ratio using distilled water as a liquid.

\section{Acknowledgments}

The first author acknowledges the financial support of this research project by the China Scholarship Council (CSC) under contract Nr.20170 8080130. Furthermore, thanks to the guidance from Prof. Martin Sommerfeld, all colleagues in the MPS institute and Mr. Zhenbin Fan for 3D printing.

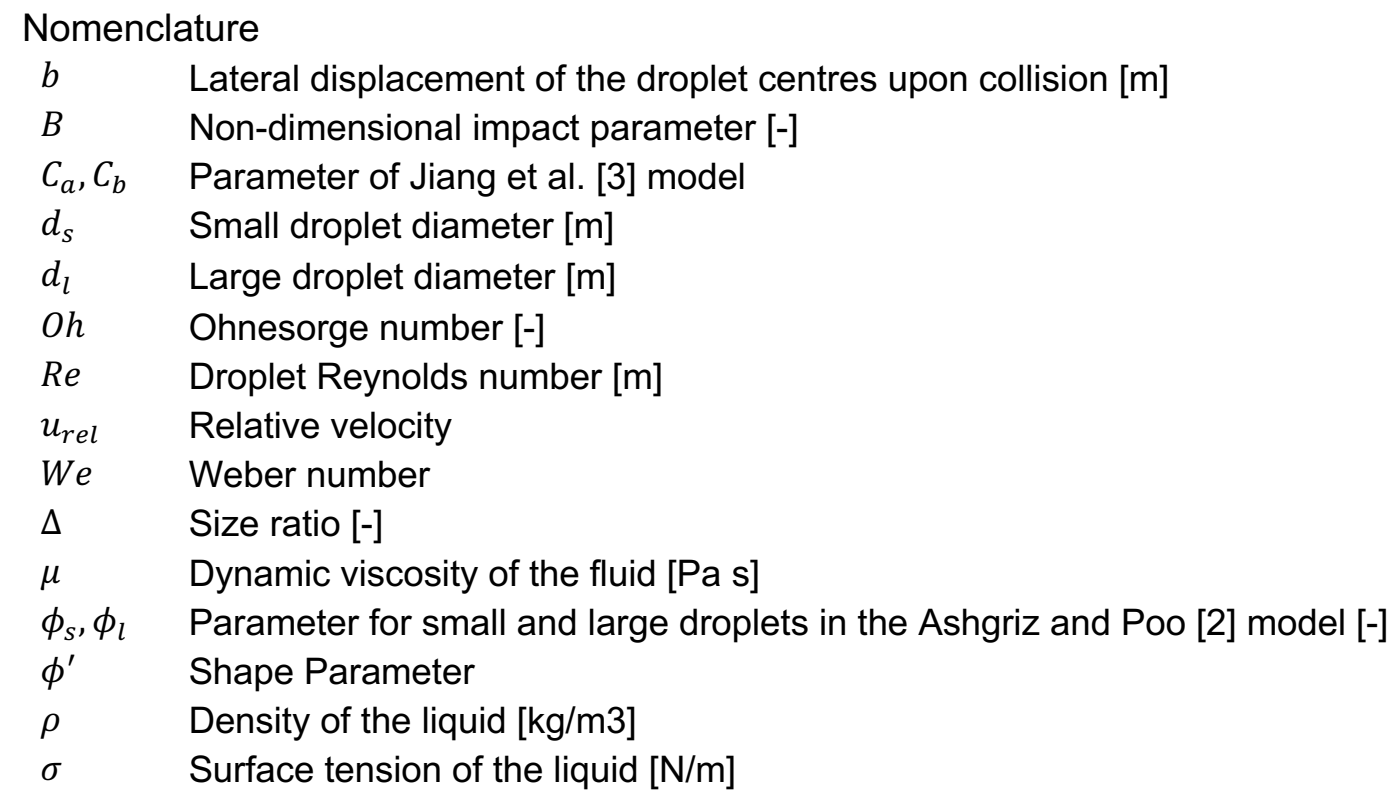




\section{References}

[1] Sommerfeld, M., and Pasternak, L., 2019, "Advances in Modelling of Binary Droplet Collision Outcomes in Sprays: A Review of Available Knowledge," International Journal of Multiphase Flow, 117, pp. 182-205.

[2] Lain, S., and Sommerfeld, M., 2020, "Influence of Droplet Collision Modelling in Euler/Lagrange Calculations of Spray Evolution," International Journal of Multiphase Flow, 132, p. 103392.

[3] Estrade, J.-P., Carentz, H., Lavergne, G., and Biscos, Y., 1999, "Experimental Investigation of Dynamic Binary Collision of Ethanol Droplets - a Model for Droplet Coalescence and Bouncing," International Journal of Heat and Fluid Flow, 20(5), pp. 486-491.

[4] Ashgriz, N., and Poo, J. Y., 1990, "Coalescence and Separation in Binary Collisions of Liquid Drops," Journal of Fluid Mechanics, 221, pp. 183-204.

[5] Qian, J., and Law, C., 1994, "Effects of Liquid and Ambient Gas Properties on Droplet Collision," Effects of Liquid and Ambient Gas Properties on Droplet Collision, American Institute of Aeronautics and Astronautics.

[6] Jiang, Y. J., Umemura, A., and Law, C. K., 1992, "An Experimental Investigation on the Collision Behaviour of Hydrocarbon Droplets," Journal of Fluid Mechanics, 234, pp. 171-190.

[7] Gotaas, C., Havelka, P., Jakobsen, H. A., Svendsen, H. F., Hase, M., Roth, N., and Weigand, B., 2007, "Effect of Viscosity on Droplet-Droplet Collision Outcome: Experimental Study and Numerical Comparison," Physics of Fluids, 19(10), p. 102106.

[8] Kuschel, M., and Sommerfeld, M., 2013, "Investigation of Droplet Collisions for Solutions with Different Solids Content," Experiments in Fluids, 54(2).

[9] Sommerfeld, M., and Kuschel, M., 2016, "Modelling Droplet Collision Outcomes for Different Substances and Viscosities," Experiments in Fluids, 57(12).

[10] Sui, M., Sommerfeld, M., and Pasternak, L., 2019, "Modelling the Occurrence of Bouncing in Droplet Collision for Different Liquids."

[11] Rabe, C., Malet, J., and Feuillebois, F., 2010, "Experimental Investigation of Water Droplet Binary Collisions and Description of Outcomes with a Symmetric Weber Number," Physics of Fluids, 22(4), p. 047101.

[12] Foissac, A., Malet, J., and Mimouni, S., 2010, Binary Water Droplet Collision Study in Presence of Solid Aerosols in Air.

[13] Pasternak, L., and Sommerfeld, M., 2017, "Experimental investigation of size effects in colliding droplet," Ilass Europe. 28th european conference on Liquid Atomization and Spray Systems, Editorial Universitat Politècnica de València, pp. 710-715.

[14] BRADSKI, G., 2000, “The OpenCV Library,” Dr Dobb's J. Software Tools, 25, pp. 120-125.

[15] Brazier-Smith, P. R., Jennings, S. G., and Latham, J., 1972, "The Interaction of Falling Water Drops: Coalescence," Proc. R. Soc. Lond. A, 326(1566), pp. 393-408. 